\title{
Excretion of hippuric acid and $m$ - or $p$-methylhippuric acid in the urine of persons exposed to vapours of toluene and $m$ - or $p$-xylene in an exposure chamber and in workshops, with specific reference to repeated exposures
}

\author{
MASANA OGATA, YOSHIKO TAKATSUKA, and \\ KATSUMARO TOMOKUNI \\ (with the technical assistance of KOZUE MUROI) \\ Department of Public Health, Okayama University Medical School, Okayama, Japan
}

\begin{abstract}
Ogata, M., Takatsuka, Y., and Tomokuni, K. (1971). Brit. J. industr. Med., 28, 382-385. Excretion of hippuric acid and $m$-or $p$-methylhippuric acid in the urine of persons exposed to vapours of toluene and $m$-or $p$-xylene in an exposure chamber and in workshops, with specific reference to repeated exposures. Four male volunteers were exposed to 200 p.p.m. of toluene for five one-hour periods separated by one-hour intervals. The excretion curve of hippuric acid showed multi-peaks, and almost concided with a theoretical curve previously described. The fraction of the toluene absorbed which was accounted for as hippuric acid was only slightly lower than after a single exposure.

In a paint spraying shop exposure was measured both from the concentrations of toluene in the air by a Kitagawa detector and from the excretion of urinary hippuric acid. The results were in general agreement, with a correlation coefficient of 0.67.

Urinary hippuric acid and methylhippuric acid were determined on urines from two workers in a shipbuilding yard who used paint thinned with toluene and xylene. The concentrations of the acids varied from day to day depending on the kind and the duration of work. From the concentrations found the mean concentrations to which the workers were exposed were calculated as a fraction of the maximum allowable concentration (M.A.C.). One worker was, on this evidence, exposed to more than the combined M.A.C. on three days out of six.
\end{abstract}

In a previous report (Ogata, Tomokuni, and Takatsuka, 1970) the relationship between the concentrations in air of toluene or $m$ - and $p$-xylene in an exposure chamber and the quantities of hippuric acid or $m$ - and $p$-methylhippuric acids in the urine of volunteers was described. In this experiment the air concentrations of toluene and xylene were kept constant. In the workshop, however, workers are exposed to toluene of markedly varying concentra- tions. In order to clarify differences in the excretion rates after single and multiple exposures, volunteers were exposed to toluene five times, and the total excretion of hippuric acid was determined and compared with that following a single exposure (Ogata et al., 1970). In the workshop, where workers were also exposed repeatedly to toluene in the course of their work, we determined the average toluene concentrations in air every 15 minutes, and compared 
the quantities absorbed as calculated from the exposure and the workers' urinary hippuric acid.

Urinary hippuric and methylhippuric acids were also estimated and evaluated in workers using paint thinner containing toluene and xylene.

\section{Methods}

\section{Subjects}

Experiments using the exposure chamber Experiments were carried out with four volunteers, research workers in our laboratory, of average age 37 years. They were exposed to 200 p.p.m. of toluene for five periods of one hour, with one hour's intermission between each.

Workers using toluene In an automobile factory, workers first sprayed a paste dissolved in toluene on the ceiling of drivers' cabins and then pasted plastic boards on them. Environmental toluene concentrations were measured in the spraying shop, both inside the drivers' cabins and outside them where the workers moved from one room to the next. The average toluene concentration to which the workers were exposed was calculated from toluene concentrations and the time spent at each place.

Workers using thinner In a shipbuilding yard two workers sprayed or painted manually paint onto the walls of cabins and bottoms of ships.

\section{Urine specimens}

Urine samples were taken from volunteers before entering and after leaving the exposure chamber, and $\mathbf{3 0}$ minutes after entering the chamber. The urine continued to be collected for about one day after exposure. From workers' urine specimens were collected from 6.00 to 8.30 a.m., again from 8.30 a.m. to 12 noon, and finally from noon to $4.30 \mathrm{p} . \mathrm{m}$. and were classified as urine from before, during, and after working respectively.

Hippuric and methylhippuric acids were determined as described previously (Ogata, Tomokuni, and Takatsuka, 1969). Some results were expressed in milligrammes per minute.

Determination of toluene concentrations in the air In an artificial exposure chamber the vapour concentration in the chamber was determined every half hour by a gas chromatograph with a flame ionization detector (Hitachi Co. Ltd. K-53) and was also checked by the Kitagawa detector tube every 15 minutes. The average of the concentrations determined by gas chromatography was taken as correct. It was constant to within $\pm 9 \%$. The quantity of hippuric acid which should appear in the urine was calculated by the formula given previously (Ogata et al., 1969) on the assumption that all the toluene absorbed was converted and excreted. For the determination of the toluene concentrations in the automobile factory a Kitagawa detector tube was used.

\section{Determination of thinner ingredients}

The paint was distilled under reduced pressure, and the distillates obtained were analysed by gas chromatography using the internal standard method.
Results
Repeated toluene exposures
The excretion curve of hippuric acid in the urine of volunteers exposed in an exposure chamber to 200 p.p.m. toluene for five periods of one hour with onehour intervals is shown in Figure 1. The excretion curve showed multi-peaks. The apex of each was at the time when the subject left the chamber. As the half-period of excretion of hippuric acid is 2.9 hours, the more often the exposure was repeated, the higher the observed peak. The curve linking all the peaks and that linking all the troughs was of the exponential type described theoretically by Roach (1966). The total excretion of hippuric acid calculated by the equation given previously (Ogata et al., 1970) was equivalent on a molar basis to about $63 \%$ of the toluene inhaled in this experiment, as against about $68 \%$ of that inhaled when the air concentration was constant (Ogata et al., 1970). The difference is not great, though after repeated exposure a slight decrease in the excretion was observed. This suggests that results from experiments using a single gas exposure can be useful for estimating the average concentration inhaled by workers exposed repeatedly and intermittently.

\section{The workshop}

In an automobile factory some workers sprayed paste dissolved in toluene on plastic board and carried it to drivers' cabins. Other workers sprayed the paste on the ceilings of drivers' cabins, and

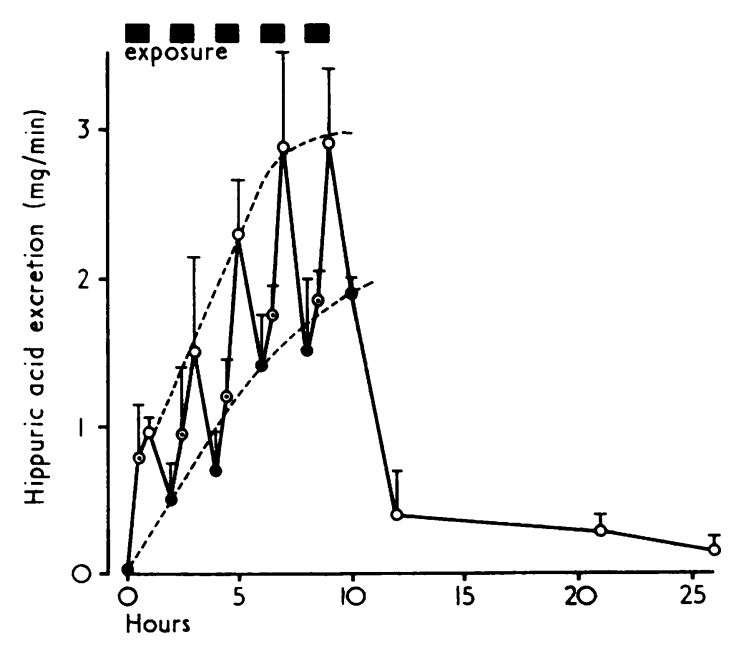

FIG. 1. Urinary excretion rates of hippuric acid in the urine of volunteers exposed to 200 p.p.m. toluene for five periods of one hour, each separated by one hour (mean + S.E.M.; - S.E.M. omitted for clarity):

urine taken just before entering chamber

$\odot$ urine taken 30 min after entering chamber

$\bigcirc$ urine taken just after leaving chamber 
pasted the plastic board on them. The average toluene concentration was 214 p.p.m. in the cabins where the workers worked for about 1.5 minutes and 3 p.p.m. in the shop outside, where workers moved from one seat to the next, and where different workers spent different lengths of time. The workers were, therefore, exposed repeatedly to alternating high and low concentrations of toluene.

The average toluene concentration to which each worker was exposed was calculated from the toluene concentration at his nose and from the duration of exposure. Figure 2 shows that these concentrations were usually in fair accordance with those calculated from the amounts of urinary hippuric acid on the basis of the results from the artificial exposure chamber. The regression line, however, deviated slightly from the theoretical line, which is at $45^{\circ}$ through the origin, and the correlation coefficient was only $\mathbf{0 \cdot 6 7}$.

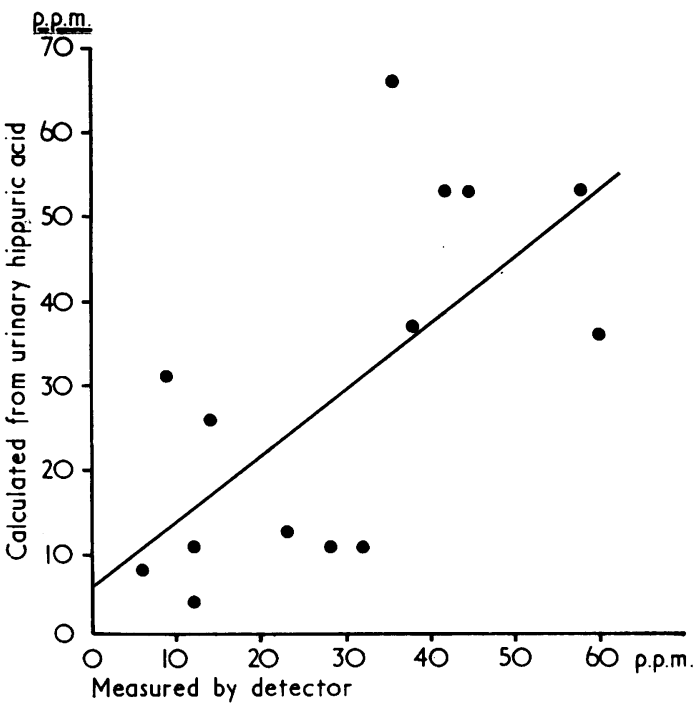

FIG. 2. Relation between the average toluene concentration measured by a Kitagawa detector and that calculated from the urinary excretion rate of hippuric acid.

$$
y=0 \cdot 78 x+6 \cdot 81, \mathrm{r}=0.67
$$

\section{Ingredients of the thinner}

The thinner used in spraying work contained $29.6 \%$ of toluene and $32.8 \%$ of $m$ - or $p$-xylene. The molar ratio of toluene to $m$-and $p$-xylene was thus 1:0.96.

One worker, $Y$, was engaged in paint spraying and manual painting and was supervised by another, I. The urinary hippuric and methylhippuric acids of $Y$ were markedly higher than those of I (Fig. 3 and Table). For $Y$, the concentrations on the days when he was engaged in paint spraying were higher than
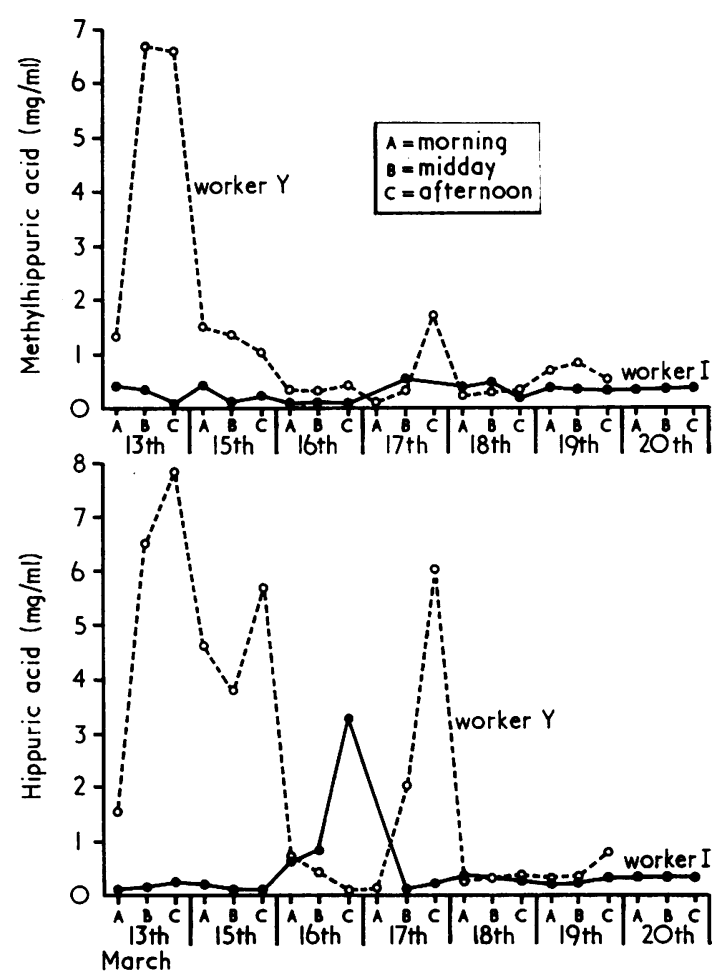

FIG. 3. Concentrations of urinary hippuric acid and methylhippuric acid in workers painting in a shipbuilding yard.

on the days when he was engaged in manual painting. The concentrations rose during the day and were maximal at the end of the work.

The molar ratio of hippuric acid to methylhippuric acid varied during a working day over the range 2.3 to 5.0 on March 13,15 , and 17, i.e., $Y$ excreted hippuric acid and methylhippuric acids in high concentrations (Table). (In this experiment, as only $2 \mathrm{ml}$ of urine per specimen were obtained, we could not measure specific gravity, so uncorrected concentrations are used.)

As maximal allowable concentrations (M.A.C.) of toluene and xylene are different-200 and 100 p.p.m. respectively (American Conference of Governmental Industrial Hygienists, 1967) - the fraction of the combined M.A.C. likely to have been present can be calculated from urinary concentrations by the following formula (Ogata et al., 1970):

Concentration of hippuric acid Mean concentration for 200 p.p.m. of toluene
$(4.56 \mathrm{mg} / \mathrm{ml})$

Concentration of methylhippuric acid

Mean concentration for 100 p.p.m. of $=$ fraction(F) $m$-xylene $(1.86 \mathrm{mg} / \mathrm{ml})$ 
TABLE

Concentrations (Mg/ML) of Hippuric Acid (HA) ANd Methylhippuric Acid (MHA) in the Urine of Workers in a Shipbuilding Yard and the Fraction (F) OF THE Combined MAC for Worker Y

\begin{tabular}{|c|c|c|c|c|c|c|c|c|c|c|c|c|}
\hline & \multicolumn{2}{|c|}{ March 13} & \multicolumn{2}{|c|}{ March 15} & \multicolumn{2}{|c|}{ March 16} & \multicolumn{2}{|c|}{ March 17} & \multicolumn{2}{|c|}{ March 18} & \multicolumn{2}{|c|}{ March 19} \\
\hline & a.m. & p.m. & a.m. & p.m. & a.m. & p.m. & a.m. & p.m. & a.m. & p.m. & a.m. & p.m. \\
\hline & $S$ & $S$ & $M \& S$ & $M$ & $M$ & $M$ & $M$ & $S$ & $R$ & $M$ & $M$ & $M$ \\
\hline $\begin{array}{l}\text { HA } \\
\text { MHA } \\
\text { F }\end{array}$ & \multicolumn{2}{|c|}{$\begin{array}{l}6 \cdot 7 \\
7 \cdot 1 \\
5 \cdot 0^{1}\end{array}$} & \multicolumn{2}{|c|}{$\begin{array}{l}1 \cdot 2 \\
4 \cdot 8 \\
2 \cdot 7^{1}\end{array}$} & \multicolumn{2}{|c|}{$\begin{array}{l}0.3 \\
0.2 \\
0.2\end{array}$} & \multicolumn{2}{|c|}{$\begin{array}{l}1 \cdot 0 \\
4 \cdot 0 \\
2 \cdot 3^{1}\end{array}$} & \multicolumn{2}{|c|}{$\begin{array}{l}0 \cdot 3 \\
0 \cdot 4 \\
0 \cdot 3\end{array}$} & \multicolumn{2}{|c|}{$\begin{array}{l}0 \cdot 6 \\
0 \cdot 5 \\
0 \cdot 4\end{array}$} \\
\hline
\end{tabular}

$S=$ spraying; $M=$ manual painting; $R=$ removing rust

March 14 was a Sunday, with no exposure

${ }^{1} \mathrm{~F}$ values over 1

Values of the fraction greater than 1 would indicate that the subject had probably been exposed to a total concentration greater than the combined M.A.C. Such values were found on March 13, 15, and 17 (Table).

\section{Discussion}

The present results on controlled exposure show that after absorption of toluene very similar fractions are accounted for as urinary hippuric acid under the conditions of the test whether exposure is continuous or intermittent. In the workers in the paint spraying shop of an automobile factory, where there was repeated exposure to varying concentrations of toluene, the average environmental concentration measured with a Kitagawa detector agreed fairly well with the average concentration calculated from urinary excretion of hippuric acids on the basis of the controlled exposure experiments. In the workshop the relationship between the measured toluene concentration in the air to which each worker was exposed and that inhaled as estimated from excretion was only a rough one, because it is difficult to measure concentrations in air accurately enough by Kitagawa detection tubes at short intervals, and also because the urinary excretion by individuals is rather variable. The results overall, however, indicate that repeated exposure to toluene does not significantly modify the metabolic fate of toluene.

In the shipbuilding yard the urinary concentration of hippuric acid or methylhippuric acid varied from day to day. It is likely that in the yard the workers engaged in paint spraying inhaled more toluene than those engaged in manual painting, and that among those spraying exposure varied as they changed positions from day to day, and the evaporation velocities of toluene and xylene were modified by the conditions of spraying at each place.

The amounts of hippuric acid and methylhippuric acid excreted will indicate the amount of toluene and $m$ - or $p$-xylene retained by workers, and from the amounts of each excreted the exposure can be calculated as a fraction of the combined M.A.C. As, however, thinner contains other organic compounds, such as $o$-xylene, butanol, ethyl acetate, etc., which are also volatile and toxic, the value of urinary hippuric acids as a test of exposure to toxic vapours underestimates the hazard from inhaling thinner.

\section{References}

American Conference of Governmental Industrial Hygienists (1967). Threshold limit values for 1967 , adopted at the 29th Annual Meeting of A.C.G.I.H., Chicago, Illinois. Industr. Hyg. Dig., 31, No. 9 (Sept.) I-IX.

Ogata, M., Tomokuni, K., and Takatsuka, Y. (1969). Quantitative determination in urine of hippuric acid and $m$ - or $p$-methylhippuric acid, metabolites of toluene and $m$ - or p-xylene. Brit. J. industr. Med., 26, 330-334.

,,$---(1970)$. Urinary excretion of hippuric acid and $m$ - or $p$-methylhippuric acid in the urine of persons exposed to vapours of toluene and $m$ - or $p$-xylene as a test of exposure. Brit. J. industr. Med., 27, 43-50.

Roach, S. A. (1966). A more rational basis for air sampling programs. Amer. industr. Hyg. Ass., J., 26, 1-12.

Received for publication October 21, 1970. 